\title{
A CONTRIBUTION TO THE THEORY OF SOLUTION
}

BY GEOFFREY MARTIN

\section{Sphere of Molecular Activity}

Consider any one molecule $m$ inside a fluid. It is surrounded by a group of molecules. If we take all these molecules which lie within a sphere of extremely small radius whose centre is $m$, there is a special action exerted on $m$ by each molecule within this sphere, those molecules nearest to $m$ exerting a more powerful action than those near the surface of the sphere. Beyond a certain distance $\epsilon$ from $m$ these special actions are assumed to be insensible. This length $\epsilon$ is the radius of the aforesaid sphere, called the sphere of molecular activity.

$\epsilon$ is an arbitrary distance, which decreases as we increase the motion of the molectles; for the molecules of a fluid are in incessant motion and in consequence of this motion are in a state of incessant flux to and fro about every point in the liquid so that every molecule remains on an average under the influence of another for a short time $t$, as they pass each other in the liquid, $-t$ depending on the velocity of the molecule, and the greater is the velocity the smaller is $t$. If, now, in the time $t$, during which the molecule $A$ is under the influence of the molecule $B$, the distance $\epsilon$ which separates them is so wide that the force which A exerts has in this time no appreciable influence in curving the path of $B$, we assume that this distance $\epsilon$ is the required limit of the sphere of molecular activity.

If, now, the velocity of the molecules be increased, the average time $t$, during which one molecule is under the action of another, will diminish; and therefore; in order that the force $F$ which the one molecule must exert on the other in order to produce an appreciable curvature in this shorter time $t$, the molecules must now approach each other closer than previously, for this will make the attractive force stronger, and so neutralize the smaller time for which the forces act. It is clear, therefore, that $\epsilon$ decreases, and $F$ increases as the velocities of the molecules increase, and vice-versa.

That is to say, ' $\epsilon$ decreases and $F$ increases as the tempera- 
ture increases; and conversely $\epsilon$ increases and $F$ decreases as the temperature of the liquid falls.

It is owing to this fact (as we shall prove later) that the solubility increases with the temperature and decreases with fall of temperature.

2. Every Dissolved Molecule in a Liquid is Surrounded by a Vacuum

Consider now a liquid whose molecules are all of the same kind. Then the molecules will on the average be arrariged symmetrically about every point within it. If, however, we introduce into the liquid a foreign molecule $m$ (see Fig. I), the foreign molecule will form a centre of disturbance and destroy the symmetrical structure of the liquid about it. For, consider any molecule $M_{I}$ of the liquid. Describe about $M_{1}$ the sphere of molecular activity $\mathrm{ABC}$ of radius $\epsilon$. Now take any other molecule $\mathrm{M}_{2}$ of the liquid which lies on this sphere of molecular activity, and describe about $\mathrm{M}_{2}$ its sphere of molecular activity ABD. Then $M_{2}$ and every molecule beyond $M_{2}$ is unaffected by $\mathrm{M}_{1}$ and vice versa. Let now the molecule $\mathrm{M}_{1}$ be replaced by a foreign molecule which is different in nature from the other surrounding molecules of the liquid. Two cases must be considered :

Case $I$. When the molecules of the liquid attract each other more strongly than they attract $\mathrm{M}_{1}$.

Case II. When the molecules of the liquid attract each other less strongly than they attract $\mathrm{M}_{1}$.

Case I.-Consider any molecule $m$ (see Fig. I) in the interval

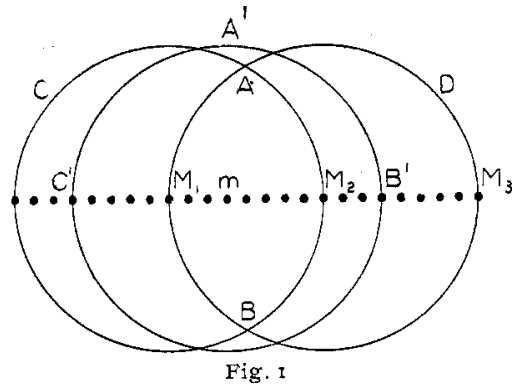

between $\mathrm{M}_{1}$ and $\mathrm{M}_{2^{\prime}}$. Describe about $m$ its sphere of molecular activity $\mathrm{A}^{\prime} \mathrm{B}^{\prime} \mathrm{C}^{\prime}$. Then $m$ is pulled in the direction $m \mathrm{~B}^{\prime}$ by the 
united action of the molecules which lie in the line $m \mathrm{~B}^{\prime}$. Similarly $m$ is pulled in the contrary direction by the action of the molecules which lie in the line $m \mathrm{C}^{\prime}$. But since the molecule $\mathrm{M}_{1}$ exerts a weaker pull on $m$ than it could exert were it the molecule of the liquid it has displaced, it is clear that there will be a resultant force exerted on $m$ in the direction $m \mathrm{~B}^{\prime}$; and so $m$ will tend to move away from $\mathrm{M}_{1}$ until it passes beyond the sphere of molecular activity ABC. Every molecule of the liquid within the sphere of molecular activity of $\mathrm{M}_{1}$ will be acted upon by a resultant force tending to direct it away from $M_{1}$. A sort of vacuum bubble will therefore form around $M_{1}$, and $M_{1}$ will continually travel about the liquid in this vacuum bubble; for as it moves forward the molecules of the liquid will be drawn away from before it, so that the dissolved molecule will possess great freedom of movement, behaving, in fact, as if it were in a vacuum.

This puts us in a position to understand why van't Hoff's law of osmotic pressure should hold true. Van't Hoff believes that a substance dissolved in a liquid exerts the same pressure on the walls of the vessel as were the solvent imagined removed and the dissolved substance existed in a gaseous state in the space occupied by the liquid. This view has been seriously questioned by many authors of great mathematical repute. For example, Prof. Oskar Meyer remarks regarding this point ${ }^{3}$ : "... Osmotic pressure is not one of the phenomena which the kinetic theory of gases has to explain. I will also not conceal that I do not think van't Hoff's views of the kinetic nature of osmotic pressure to be correct. For osmose does not arise from the kinetic pressure of the dissolved substance, but from quite different forces. ..."

These authors have, however, entirely neglected the fact that all kinds of molecules do not exert attractive forces of the same intensity. For example, the molecules of carbon exert an enormous attractive force on each other, as is shown by the extraordinary hardness and involatility of certain forms of carbon. Oxygen, helium, nitrogen molectles, however, exert but a feeble attractive force on each other.

${ }^{1}$ Kinetic Theory of Gases, English Translation (1 899), p. 367 . 
Case II.-Here the molecules of the liquid attract $M_{1}$ more strongly than they attract themselves. In this case the molecules of the liquid combine with $\mathrm{M}_{1}$, and the combination will proceed until the molecules of the liquid attract each other more strongly than they attract the new compound, so that the case reduces to Case $I$, but with this difference-the new unit is not the original molecule $\mathrm{M}_{1}$ but the compound formed between $\mathrm{M}_{1}$ and the liquid molecules. Traces of such compounds are very often met with in solution.

In this connection it should be observed that when a molecule adds on atoms or molecules to itself, the intensity of the external molecular attraction it exerts usually diminishes. For example in organic chemistry saturated compounds are more volatile than unsaturated compounds of the same molecular weight. In inorganic chemistry, compounds of a high valency grade are more volatile than compounds of the same molecular weight but of a lower valency grade. Unstable compounds are, in general, more volatile and fusible than stable compounds of the same molecular weight, and the addition of atoms to a molecule decreases its stability. This matter is fully disctissed in an earlier paper, to which the reader is referred.

3. Magnitude of the Repulsive Force $R$ Exerted on $m$ at any Distance d from $\mathrm{M}_{1}$

The repulsive force $\mathrm{R}$ exerted on $m$ (see Fig. I) at a distance $d$ from $\mathrm{M}_{1}$ is clearly the difference in the intensity of the force which a molecule of the liquid if at $\mathrm{M}_{1}$ would exert on $m$, and the force which the foreign molecule M actually exerts. (Compare Archimedes Principle in Hydrostatics.)

If at a distance $d$ a liquid molecule exerts a force $\mathrm{F}(d)$, and if at a distance $d$ a foreign molecule exerts a force $f(d)$, then the repulsive force $\mathrm{R}$ acting on $m$ at a distance $d$ is

$$
\mathrm{R}=\mathrm{F}(d)-f(d) .
$$

If we assume (as is always done by writers on molecular physics-principally, by the way, because it is convenient) that the law with which the force decays with the distance is the same for all kinds of molecules, the equation (I) assumes a

${ }^{1}$ Chem. News, 89, 24I (1904). 
much simpler form; for $f(d)$ now becomes $k \cdot \mathrm{F}(d)$, where $k$ is a constant less than unity. Hence

$$
\mathbf{R}=(\mathbf{r}-k) \cdot \mathbf{F}(d) .
$$

If the foreign molecules exert forces of the same intensity as the liquid molecules, then in (I) $f(d)=.\mathrm{F}(d)$ and $\mathrm{R}=0$; and in (a), $k=\mathrm{I}$ and $\mathrm{R}=\mathrm{O}$. This is the case when the compounds are chemically similar, as I will show in a paper which will be published later.

\section{Limit of Solubility}

Consider the molecule $\mathrm{B}$ of the foreign substance traveling in the middle of a "vacuum bubble" in the interior of the

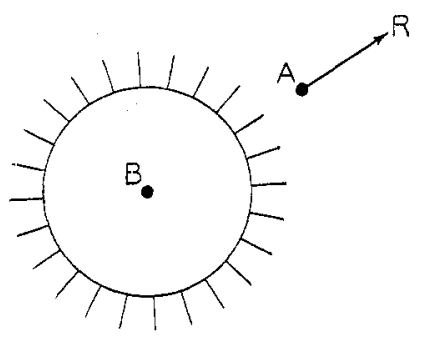

Fig. 2.

liquid. Suppose now a molecule $A$ of the liquid to move towards B. Then as it approaches the surface of the bubble it will begin to be acted upon by a force tending to pull it backwards into the liquids. Consider now the two foreign molecules

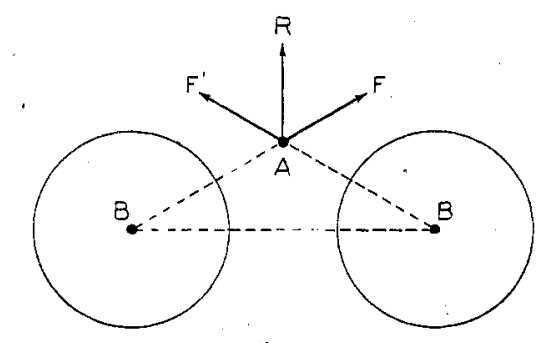

Fig. 3.

$\mathrm{B}, \mathrm{B}^{\prime}$ to be close together, and consider a molecule $\mathrm{A}$ of the liquid situated as in the figure. Then $\mathrm{A}$ will be attracted away from $\mathrm{B}$ in the direction $\mathrm{AF}$; and away from $\mathrm{B}^{\prime}$ in the direction $\mathrm{AF}^{\prime}$. Consequently, there will be a resultant force $R$ acting on $A$ tending to drive it away from the line $\mathrm{B}, \mathrm{B}^{\prime}$ joining the molecules 
$\mathrm{B}, \mathrm{B}^{\prime}$. Consequently, the two molecules $\mathrm{B}, \mathrm{B}^{\prime}$ will tend to draw together in the liquid as soon as B comes within the sphere of attraction of $\mathrm{B}^{\prime}$.

Now before the molecule $A$ of the liquid" will be repelled away from the line $\mathrm{BB}^{\prime}$, the force exerted on $\mathrm{A}$ must be of an appreciable magnitude $F$ (see Section I). Consequently, so long as the molecules $\mathrm{B}, \mathrm{B}^{\prime}$ are at such a distance apart that the force exerted on $\mathrm{A}$ is smaller than $\mathrm{F}, \mathrm{A}$ will not tend to move away from the line $\mathrm{BB}^{\prime}$, and so $\mathrm{B}$ and $\mathrm{B}^{\prime}$ will not tend to draw together.

Whence we arrive at the cause of the limited solubility of one body in another. A liquid can only dissolve so many molecules that the average distance between any two of these molecules is greater than the critical distance, whereat the repulsive force $R$ reaches a certain minimum value $F$ depending upon the temperature, molecular weight, and nature of the liquid. If a greater number of molecules $\mathrm{B}, \mathrm{B}^{\prime}$ are dissolved in the liquid, the molecules will draw together, and separate out until the average distance between the dissolved molecules becomes greater than this critical distance.

\section{Reason why the Solubility Increases with the Temperature}

The minimum repulsive force $F$ (see Section I) increases as the kinetic energy of the molecules of the liquid increases; and conversely diminishes as the kinetic energy of the molecules diminishes. Consequently the molecules of the liquid can approach nearer at high temperatures than at low temperatures without being repelled; so that $B$ and $B^{\prime}$ can approach nearer. together without being drawn together when the liquid is hot than when it is cold; so that in general the solubility of the molecules of $\mathrm{B}$ in the liquid $\mathrm{A}$ must increase or decrease as the temperature increases or decreases.

6. The Cause why Chemically Similar Substances are Miscible in all Proportions, and Chemically Unlike Substances usually Unmiscible

The repulsive force (see Section 3 ) exerted on a molecule is $\mathrm{R}=\mathrm{F}(d)-f(d) \quad$ or $\quad \mathrm{R}=(1-k) \cdot \mathrm{F}(d)$.

If the two compontuds are chemically similar, in general $j(d)$ $=\mathrm{F}(d) ;$ or $k=\mathrm{I}$. Then in this case $\mathrm{R}=0$, and the 
minimum repulsive force $\mathrm{F}$ is never attained, no matter how closely A approaches to B. Consequently, the two molecules B and $B^{\prime}$ do not tend to draw together and separate out. Hence the molecules $B$ are in this case miscible in all proportions among the molecules of $\mathrm{A}$. If, however, the two substances are not chemically similar, then in general $\mathrm{R}$ has always a finite magnitude greater than $\mathrm{F}$ and so the two liquids will separate out into two layers when the molecules of $B$ assume more than a definite average distance apart in the manner previously described.

In hydrostatics a cork tends to be expelled out of the water because the water it displaces weighs more than the cork; whereas a body of the same density as water does not tend to separate out from water. Similarly, a chemically dissimitar molecule tends to be expelled from among the intermolecular interstices of a liquid because the force it exerts on the surrounding molecules is less than the force which the molecule of the liquid it displaces exerts, and a chemically similar molecule does not tend to be expelled out of the molecular interstices of the liquid because the forces it exerts are of the same magnitude as those of the liquid molecule it has displaced.

7. Why Molecules so often Dissociate when they Pass into Solution

A molecule $A$ in the interior of a liquid is surrounded equally on every side by other molecules, each of which exert an attractive force on $\mathrm{A}$. The molecule of $\mathrm{A}$, then is subjected to an outward tension directed equally in all'directions. This tension has been called by Lord Rayleigh ${ }^{i}$ the intrinsic pressure of the liquid. If the molecule $\mathrm{A}$ is not of a rigid enough constitution, it would tend to expand out in every direction under the action of these attractive forces. "This is the reason why so many molecular systems, when they pass into solution, decompose under this strain into their constituent atoms or ions. It is only when the internal attractive force holding the constituent parts of the molecule together is greater than the "internal tension" of the liquid that the molecule holds together undissociated in the liquid.

University of Kiel Oct. 19, 1904.

${ }^{1}$ Phil. Mag. October, 1890. 